\title{
Evaluación del seguimiento farmacoterapéutico en pacientes ingresadas con alto riesgo obstétrico en el Hospital Universitario de Guayaquil
}

Evaluation of the pharmacotherapeutic follow-up in patients admitted with high obstetric risk in the University Hospital of Guayaquil

\author{
Betsy Fajardo Vélez \\ Frella Soraya García Larreta \\ Luis Armando Arias Duque
}

Fecha de recepción:03 de noviembre del 2020

Fecha de aceptación:21 de diciembre del 2020 


\title{
Evaluación del seguimiento farmacoterapéutico en pacientes ingresadas con alto riesgo obstétrico en el hospital universitario de Guayaquil
}

\author{
Evaluation of the pharmacotherapeutic follow-up in patients admitted with high \\ obstetric risk in the university hospital of Guayaquil
}

Betsy Fajardo Vélez ${ }^{1}$, Frella Soraya Garcia Larreta ${ }^{2}$, Luis Armando Arias Duque ${ }^{3}$

Como citar: Fajardo, B., García, F., Arias, L., (2021). Evaluación del seguimiento farmacoterapéutico en pacientes ingresadas con alto riesgo obstétrico en el Hospital Universitario de Guayaquil, Revista Universidad de Guayaquil. 132 (1). 48-56. doi: https://doi.org/10.53591/rug.v133i2.1382

\section{RESUMEN}

El uso de medicamentos durante la etapa de gestación se ha vuelto cada vez más frecuente, esto a razón de que durante este periodo ocurren diversos cambios fisiológicos que podrían afectar las enfermedades preexistentes o inducir a trastornos específicos del embarazo, por lo que es necesario promover una farmacovigilancia activa a través del seguimiento farmacoterapéutico. El presente documento es el resultado de la investigación realizada en el Hospital Universitario de Guayaquil a pacientes que fueron ingresadas con alto riesgo obstétrico en el periodo de enero a junio del 2021, donde se pudo catalogar los diferentes diagnósticos de acuerdo con el rango de edad, nivel de riesgo y problemas relacionados con la medicación suministrada durante la estancia hospitalaria. El objetivo principal de la investigación es evaluar la efectividad de la atención farmacéutica a través del seguimiento farmacoterapéutico durante el periodo establecido para el estudio. El trabajo se realizó mediante el servicio de dispensación por dosis unitaria, utilizando como instrumentos principales: el perfil farmacoterapéutico registrado diariamente durante la permanencia en el servicio de hospitalización de las pacientes, el registro de intervenciones farmacéuticas y la revisión de historias clínicas. El total de perfiles elaborados fue de 144 y los Problemas Relacionados con medicamentos (PRM) detectados fueron 82, siendo el de mayor frecuencia el PRM 1 (54.87\%) seguido del PRM 5 (15.85\%); se determinó que el rango de edad de mayor incidencia en cuanto a riesgo obstétrico es de 25 a 30 años con el $26.38 \%$ de los riesgos obstétricos encontrados.

Palabras Clave: Seguimiento farmacoterapéutico, Problemas Relacionados con Medicamentos, Atención farmacéutica.

\footnotetext{
${ }^{1}$ Magíster En Farmacia, Mención Farmacia Clínica, Universidad de Guayaquil, Ecuador. Código Orcid: https://orcid.org/0000-0001-6510-292X,Correo electrónico: betsy.fajardov@ug.edu.ec

${ }_{2}^{2}$ Magister en Diseño Curricular, Docente de la Universidad de Guayaquil, Ecuador. Código Orcid: https://orcid.org/0000-0002-5893-5939 , Correo electrónico: soraya.garcial@ug.edu.ec

${ }_{3}^{3}$ Magister en Diseño Curricular, Docente de la Universidad de Guayaquil, Ecuador. Código Orcid: https://orcid.org/0000-0002-4636-7954 , Correo electrónico: luis.ariasd@ug.edu.ec
} 


\begin{abstract}
The use of drugs during pregnancy has become increasingly frequent, this is because during this period various physiological changes occur that could affect pre-existing diseases or induce specific disorders of pregnancy, so it is necessary to promote a Active pharmacovigilance through pharmacotherapeutic monitoring. This document is the result of the research carried out at the Guayaquil University Hospital on patients who were admitted with high obstetric risk in the period from January to June 2021, where the different diagnoses could be classified according to the age range, level of risk and problems related to the medication provided during the hospital stay. The main objective of the research is to evaluate the effectiveness of pharmaceutical care through pharmacotherapeutic follow-up during the period established for the study. The work was carried out through the unit dose dispensing service, using as main instruments: the pharmacotherapeutic profile recorded daily during the stay in the hospitalization service of the patients, the registration of pharmaceutical interventions and the review of medical records. The total of profiles prepared was 144 and the Drug Related Problems (DRP) detected were 82, the most frequent being DRP 1 (54.87\%) followed by DRP 5 (15.85\%); It was determined that the age range with the highest incidence in terms of obstetric risk is between 25 and 30 years, with $26.38 \%$ of the obstetric risks found.
\end{abstract}

Keywords: Marketing. Pharmacotherapeutic follow-up, drug-related problems, Pharmaceutical Care. 


\section{INTRODUCCIÓN}

Los medicamentos constituyen una de las herramientas terapéuticas más utilizadas en la actualidad, es por ello que su acceso, utilidad y resultados proporcionados deben ser mejorados, ya que el uso inadecuado puede producir problemas de salud(De et al., n.d.), conocidos como Problemas Relacionados con Medicamentos (PRM) los cuales se han definido como un evento o circunstancia que involucra la terapia con drogas que real o potencialmente interfiere con los resultados de salud deseados (Calvo-Salazar et al., 2018). Esta es una demarcación general que puede abarcar distintos términos que se refieren a la seguridad de los medicamentos, como eventos relacionados con los mismos, reacciones adversas y errores de medicación (Ferrández et al., 2019).

Es bien conocido que varios problemas relacionados con medicamentos se desarrollan en la atención primaria, donde los pacientes suelen ser un participante activo en la revisión de medicamentos, mientras que otros PRM solo podrían identificarse en base a las historias clínicas de los pacientes que se encuentran hospitalizados, donde frecuentemente se asocia a las reacciones adversas a medicamentos como el PRM de mayor significancia clínica, no obstante, es importante mencionar que no todas las categorías de PRM están asociadas a este resultado clínico (Kjeldsen et al., 2017) (EY et al., 2016)

Se estima que aproximadamente el $5 \%$ de todos los ingresos hospitalarios se atribuyen a reacciones adversas a medicamentos, además de ciertos factores como el tratamiento subterapéutico, sobredosis y otros eventos adversos que son causas potenciales de hospitalizaciones que pueden ser prevenidas (Ravn-Nielsen et al., 2018).

Un estudio realizado por farmacéuticos clínicos de un hospital de tercer nivel en Brasil permitió detectar 170 problemas relacionados con medicamentos, que tenían el potencial de causar daño en los pacientes y otros 50 eventos adversos inevitables, esta intervención demostró ser un método efectivo no solo para la detección de PRM, sino también para la prevención de eventos adversos, lo que contribuye a mejorar la seguridad del paciente (Martins et al., 2019).

Para los sistemas de salud, la seguridad del paciente es considerado un significativo indicador de la calidad asistencial, y su mejoramiento representa una prioridad para los servicios sanitarios (Giménez-Júlvez et al., 2017). La seguridad de atención se enfoca en desarrollar sistemas y procesos encaminados a reducir la probabilidad de aparición de errores y disminuir sus consecuencias (Rocco \& Garrido, 2017), la mala utilización de medicamentos es en la actualidad un problema sanitario de gran relevancia, y el uso de varios grupos de fármacos durante la hospitalización se relacionan con mayor frecuencia en el desarrollo de (PRM) asociándolos a un elevado índice de morbi-mortalidad, 
prolongando la estancia hospitalaria y por ende el aumento de costos (Ferrández et al., 2019; Kjeldsen et al., 2017).

Históricamente la efectividad y los perfiles de riesgo de muchos de los medicamentos utilizados en los programas de salud pública en el pasado, han sido establecidos por la modalidad de ensayo- error y no sobre estudios epidemiológicos de acuerdo a las características de la población; en la actualidad el enfoque de la salud pública requiere que se brinde asesoramiento sobre las formas más idóneas de abordar el manejo de enfermedades, con la posibilidad de modificar la terapia de acuerdo a las necesidades individuales de cada paciente (WHO, 2006).

Actualmente se han implementado varias estrategias que permiten aumentar la seguridad en el uso de los medicamentos, como la implementación de prescripción electrónica y diversos sistemas integrados de apoyo a la decisión clínica, los cuales han contribuido a una disminución en el riesgo de errores de medicación y eventos adversos de medicamentos (Ferrández et al., 2019).

Los farmacéuticos tienen una importancia primordial en la identificación, tratamiento y prevención de (PRM) reales y potenciales utilizando métodos de prácticas de atención farmacéutica. Un (PRM) real es un evento que ya ha sido evidente en un paciente, mientras que un (PRM) potencial es un evento que aún no es evidente, pero que podría evidenciarse si el profesional farmacéutico no realiza las intervenciones pertinentes (Kanagala et al., 2016)

Para evaluar la efectividad de la atención farmacéutica a través del seguimiento farmacoterapéutico en pacientes con alto riesgo obstétrico, ingresadas en el Hospital Universitario de Guayaquil en un periodo de 6 meses (enero - junio 2021) se consideraron 144 pacientes a las que se realizó seguimiento farmacoterapéutico con la finalidad de detectar Problemas Relacionados con Medicamentos y resultados negativos asociados a la medicación, la recolección de la información se efectuó mediante el servicio de dosis unitaria, a través de los registros diarios de intervenciones farmacéuticas, perfiles farmacoterapéuticos, revisión de historias clínicas y validación de recetas de las pacientes que tuvieron una estancia hospitalaria superior a 48 horas en el servicio de Alto Riesgo Obstétrico.

\section{METODOLOGIA}

Esta investigación corresponde a un estudio observacional, que se refiere a un diseño de investigación que tiene por objetivo observar y registrar los acontecimientos sin que se pueda modificar el curso natural de estos, pueden ser realizados a través del tiempo (longitudinal) ya sea de forma prospectiva o retrospectiva (Manterola et al., 2019).

Es de tipo no experimental dado que no es posible influir sobre las variables, descriptiva, ya que brinda 
información respecto al "Que" "Como" "Cuando" y "Donde" sin considerar prioritario el "Porque"; a diferencia de otro tipo de investigaciones esta se limita únicamente a la medición y descripción de estas (Investigación Descriptiva: Características, Técnicas, Ejemplos, n.d.).

Además, se considera de tipo cuantitativa ya que se basa en la obtención de una serie de datos para el posterior análisis de los mismos, utilizando varios instrumentos estadísticos que permite estudiar la relación entre todos los datos cuantificados para conseguir una interpretación adecuada de los resultados obtenidos $(\triangle$ Investigación Cuantitativa: Qué Es y Características IE-Nquest】, n.d.).

La población considerada para el estudio fueron las pacientes a las que se les realizó seguimiento farmacoterapéutico, orientado a la detección de problemas relacionados con medicamentos en base a los criterios del Tercer Consenso de Granada, su vinculación con morbilidades y esquemas terapéuticos más utilizados en los ingresos registrados en los servicios obstétricos del Hospital Universitario de Guayaquil entre enero y junio del 2021, representados por 144 perfiles registrados.

\section{Criterios de inclusión}

Pacientes hospitalizadas con alto riesgo obstétrico (embarazadas y puérperas) con estancia hospitalaria superior a 48 horas.

\section{Criterios de Exclusión}

Pacientes con estancia hospitalaria menor a 48 horas, pacientes que soliciten de manera voluntaria y anticipada el alta médica.

El principal instrumento para el desarrollo de la investigación es el perfil farmacoterapéutico, establecido en la normativa de dosis unitaria. Además, la tabla de registros de intervenciones farmacéuticas elaborada por el personal farmacéutico del servicio de Dosis Unitaria Del Hospital Universitario De Guayaquil.

Para asegurar la confiabilidad de los datos y determinar la existencia de relación entre las variables, se aplicó el método de correlación de Pearson, que es una prueba que mide la relación estadística entre dos variables continuas, cuyos valores de correlación oscilan entre $-1 \mathrm{y}+1$, siendo un valor de 0 cuando no hay relación entre las variables, se acerca a -1 cuando la relación es inversa y a +1 cuando la relación es directa (Franklin et al., 2018). Los datos recolectados fueron procesados mediante las funciones estadísticas en Microsoft office Excel 2021.

La información será obtenida directamente de los perfiles farmacoterapéuticos, los cuales son registrados diariamente por el profesional farmacéutico durante el pase de visita del equipo multidisciplinario en el servicio de Alto Riesgo Obstétrico del Hospital Universitario de Guayaquil, en el período comprendido entre enero a junio del 2021.

Así mismo, se empleará el registro de intervenciones farmacéuticas, para reportar las novedades referentes a la utilización de medicamentos en las pacientes durante la estancia hospitalaria.

Cada perfil farmacoterapéutico debe registrarse de forma ordenada, consistente y bajo la responsabilidad del 
Químico Farmacéutico en conjunto con el equipo multidisciplinario

En el cual se recopilan los datos más relevantes del paciente relacionados con su estancia hospitalaria, tales como:

- Nombres y apellidos del paciente Sexo

- Historia clínica

- Servicio (Área de hospitalización)

- Edad

- Peso

- Soporte nutricional

- Alergias

- $\mathrm{N}^{\circ}$ de cama

- Médico tratante

- Medicamento

- Dosis

- Vía de administración

- Frecuencia

\section{DESARROLLO}

Realizada la compilación y sistematización de la información obtenida para el presente estudio se puede apreciar que el riesgo obstétrico es más frecuente en la población de pacientes embarazadas en relación a las puérperas, este resultado tiene similitud con la investigación realizada por RodríguezAngulo, 2019 quienes determinaron que el riesgo obstétrico se da en mayor proporción en las mujeres embarazadas (90\%) (RODRÍGUEZ-ANGULO et al., 2019). A diferencia de lo expresado por Montenegro Rivera 2020, cuyos resultados reflejan que el mayor riesgo obstétrico se encuentra en las pacientes puérperas, siendo este una de las principales causas de muerte materna, lo que podría estar relacionado con complicaciones prenatales no tratadas adecuadamente (Palma et al., 2020).

Con respecto a los diagnósticos que son más propensos a generar PRM, se encontró que los síndromes hipertensivos en el embarazo (preeclamsia severa, Síndrome de HELLP) son los de mayor frecuencia, seguidos de la ruptura prematura de membranas y amenazas de parto pretérmino, este resultado se correlaciona positivamente con el trabajo de investigación realizado por Aquino y cols 2019, que definen como los diagnósticos de mayor generación de PRM a, las amenazas de parto prematuro 24\%, ruptura de membranas 14\% y síndromes hipertensivos 13.2\% (Aquino \& Barboza, 2019). Por otro lado, se difiere con los resultados obtenidos en la investigación de Suarez Roque y cols 2019, en donde se identificaron como diagnósticos más propensos a generar PRM a las infecciones del tracto urinario (25\%), seguido de infecciones vaginales (20\%) y preclamsia - eclampsia (7\%) (Suraez 
Roque et al., 2019), lo cual posiblemente esté ligado a las condiciones socioeconómicas, geográficas, alimenticias, e idiosincrasia de las mujeres en estado de gestación.

De los PRM identificados se encontró que el $65 \%$ corresponden a problemas relacionados con la Necesidad (PRM1 y PRM2), siendo estos de mayor impacto, 22\% a la Seguridad (PRM5 y PRM6) y 13\% a la Efectividad (PRM3 y PRM4), estos resultados se asemejan a los obtenidos por Alejandra V. Rubiños y cols 2019 donde se determinó al PRM 1 como el de mayor incidencia con $44 \%$ seguido del PRM5 con 25 \% (Juárez \& Rubiños, 2019). Mientras que en contraparte en el artículo publicado en Pharmaceutical Care por Parody Rúa, al determinar la frecuencia de los PRM se halló que el 41,2\% fueron asociados a PRM de seguridad (5 y 6), 38.3\% a PRM de necesidad (1 y 2) y $20.6 \%$ a la efectividad (3 y 4) (Parody Rua, Elizabeth. Montaño Holguín, 2018); lo cual se puede deber a las condiciones fisiológicas de los pacientes objeto de investigación, diagnóstico y tratamiento aplicados, edad, etc.

\section{RESULTADOS}

En los 6 meses que corresponden al periodo de evaluación (Enero - Junio 2021), se obtuvieron resultados en cuanto a los indicadores considerados en la normativa de Dosis Unitaria, los mismos que se encuentran detallados en la tabla III.

Durante el semestre de evaluación, se registraron 144 perfiles farmacoterapéuticos en las pacientes que cumplieron con los criterios de inclusión, de las cuales el $94.44 \%$ corresponde a embarazadas y el $5.56 \%$ a puérperas.

Por otra parte, la tabla V detalla los 82 PRM identificados en la muestra de pacientes, los cuales se clasificaron en los 6 grupos de acuerdo con el Tercer Consenso de Granada; dando como resultado 55\% para el PRM 1, seguido del 16\% para el PRM 5, 10\% para el PRM 2, 7 \% para el PRM 3, 6\% para el PRM 4 y 6\% para el PRM 6.

Así mismo en la tabla VI se visualiza la cantidad de PRM asociados a los diferentes diagnósticos en la cual se identifica que el diagnóstico que genera mayor PRM, es la Preeclampsia severa con un total de 15 PRM (18.3\%), le sigue el diagnóstico Ruptura prematura de membranas con 14 PRM (17.1\%) y Amenaza de parto pretérmino con 9 PRM (11\%).

Dentro de los resultados obtenidos destacamos de forma cuantitativa la cantidad de pacientes que en base al diagnóstico y luego de la tabulación se agrupan por nivel de riesgo; se evidencia que existe mayor cantidad de pacientes en el grupo de 25 a 30 años con 38 pacientes, divididos para el riesgo 1 con 5 pacientes, para el riesgo 2 con 12 pacientes, y para el riesgo 3 con 21 pacientes. También se puede determinar que el riesgo 3 es el mayor riesgo identificado con 87 pacientes, en segundo lugar, está el riesgo 2 con 43 pacientes y en tercer lugar el riesgo 1 con 14 pacientes. 


\section{CONCLUSIONES}

- Se identificaron 82 PRM en pacientes hospitalizados con alto riesgo obstétrico, encontrando al PRM1, asociado a la necesidad, como el de mayor frecuencia con 55\%, seguido del PRM5 asociado a la seguridad del medicamento con $16 \%$.

- Los diagnósticos con mayor incidencia en Riesgo Obstétrico y aparición de PRM fueron las enfermedades hipertensivas con el 18,3\%; seguido de la Ruptura prematura de membranas con 17,1\%; y la anemia severa con el $12,2 \%$ de los PRM determinados.

- Se evidencio que uno de los factores determinantes en el aumento de riesgo obstétrico y aparición de PRM es la edad, estableciendo el rango de mayor frecuencia entre 25 y 30 años.

- Se demostró que la participación activa del farmacéutico influye de manera positiva en la atención integral del paciente, mediante el índice de aceptación de intervenciones farmacéuticas, mismo que fue del 99\%

\section{REFERENCIAS}

Alexakis, P., \& Siriopoulos, C. (1999). The international stock market crisis of 1997 and the dynamic relationships between asian stock markets: Linear and nonlinear Granger causality tests. Managerial Finance(25), 22-38.

AMA. (2013). American Marketing Association. Obtenido de Journal of Marketing Reseach: https://www.ama.org/publications/JournalOfMarketing/Pages/Current-Issue.aspx

Andreasen, A. (1994). Social Marketing: Its definition and Domain. Journal of Public Policy and Marketing, 13(Spring), 108-114.

Arcotel. (2018). Estadísticas Anuales Líneas Móviles. Recuperado el 26 de Enero de 2018, de Agencia de Regulación y Control de Telecomunicaciones: http://www.arcotel.gob.ec/servicio-movil-avanzado-sma/

Barboza, A. (2016). Sobre el Método de la interpretación documental y el uso de las imágines en la sociología. Sociedade e Estado, 21(2), 391-414.

Blodgett, J., Lu, L.-C., \& Rose, G. (2001). Ethical Sensitivity to Stakeholder Interest: A CrossCultrural Comparison. Journal of Academy of Marketing Science, 29(2), 190-202.

Claro. (Enero de 2018). Claro. Recuperado el 2 de Febrero de 2018, de Quienes Somos: https://www.claro.com.ec/personas/institucional/quienes-somos/

CNT-EP. (2018). La Corporación Nacional de Telecomunicaciones CNT EP. Recuperado el 2 de Febrero de 2018, de CNT: http://corporativo.cnt.gob.ec/historia-de-las-telecomunicacionesen-el-ecuador/

Drucker, P. (1984). The new meaning of corporate social responsi-bility. California Management Review, XXVI(2), 53-63.

Gómez, P. (2003). La gestión de marketing de ciudades y áreas metropolitanas: de la orientación al producto a la orientación al marketing. Cuadernos de Gestión, 3(1), 11-25.

Green Paper. (2011). Green Paper on corporate social responsibility. Recuperado el 2018 de Enero de 2018, de Europa: http://europa.eu/legislation

Hunt, S. (1983). General theories and the fundamentalexplananda of marketing. Journal of Marketing, 47(4), 9-17.

Kotler, P., \& Levy, S. (1969). Broading the concept of Marketing. Journal of Marketing, 33(January), 10-15. 
Kotler, P., \& Zaltman, G. (1971). Social marketing: An approach toplanned social change. Journal of Marketing, 35, 3-12.

Lideres. (Enero de 2014). Revista Lideres. Recuperado el 12 de Enero de 2018, de En 20 años, la telefonía móvil superó las expectativas: http://www.revistalideres.ec/lideres/20-anostelefonia-movil-supero.html.

Maignan, I., \& Ferrell, O. (2004). Corporate social responsibility and marketing: an integrative framework. Journal of the Academy of Marketing Science, 32(1), 19-23.

Miron, D., Petcu, M., \& Sobolevschi, M. (2011). Corporate Social Responsibility and sustainable competitiveness. Amfiteatru Economic Journal, A.S.E.(29), 163-180.

Ramos, J., \& Periáñez, I. (2003). Delimitación del Marketing con Causa o Marketing Social Corporativo mediante el análisis de empresas que realizan acciones de responsabilidad social. Cuadernos de Gestión, 3(1), 65-82.

Telefónica S.A. (2018). Movistar. Recuperado el 3 de Febrero de 2018, de Historia de Movistar: https://www.movistar.com.ec/

Torres, B. (2006). Metodología de la Investigación. Mexico D.F: Pearson.

Vaaland, T., Heide, M., \& Grønhaug, K. (2008). Corporate social responsibility: investigating theory and research in the marketing context. European Journal of Marketing, (9/10), 927953.

Vázquez, M. (2011). Investigación en educación matemática: objetivos, cambios, criterios, método y difusión. Education Siglo XXI, 29(2), 173-198.

Vicente, M. Azucena; Mediano, Lucía; . (2002). Propuestas para una segmentación estragégica del mercado ecológico . Cuadernos de Gestión, 2(1).

WBCSD. (2012). World Business Council for Sustainable Development,. Recuperado el 15 de Enero de 2018, de . Corporate social responsibility: Meeting changing expectations [pdf].: http://www.wbcsd.org 